\title{
ORIGINAL RESEARCH \\ How Do Coil Configuration and Packing Density Influence Intra-Aneurysmal Hemodynamics?
}

\author{
H.G. Morales \\ M. Kim \\ E.E. Vivas \\ M.-C. Villa-Uriol \\ I. Larrabide \\ T. Sola \\ L. Guimaraens \\ A.F. Frangi
}

\begin{abstract}
BACKGROUND AND PURPOSE: Endovascular coiling is a well-established therapy for treating intracranial aneurysms. Nonetheless, postoperative hemodynamic changes induced by this therapy remain not fully understood. The purpose of this work is to assess the influence of coil configuration and packing density on intra-aneurysmal hemodynamics.
\end{abstract}

\begin{abstract}
MATERIALS AND METHODS: Three 3D rotational angiography images of 3 intracranial aneurysms before and after endovascular coiling were used. For each aneurysm, a 3D representation of the vasculature was obtained after the segmentation of the images. Afterward, a virtual coiling technique was used to treat the aneurysm geometries with coil models. The aneurysms were coiled with 5 packing densities, and each was generated by using 3 coil configurations. Computational fluid dynamics analyses were carried out in both untreated and treated aneurysm geometries. Statistical tests were performed to evaluate the relative effect of coil configuration on local hemodynamics.
\end{abstract}

\begin{abstract}
RESULTS: The intra-aneurysmal blood flow velocity and wall shear stress were diminished as packing density increased. Aneurysmal flow velocity was reduced $>50 \%$ due to the first inserted coils (packing density $<12 \%)$ but with a high dependency on coil configuration. Nonsignificant differences $(P>.01)$ were found in the hemodynamics due to coil configuration for high packing densities (near $30 \%$ ). A damping effect was observed on the intra-aneurysmal blood flow waveform after coiling.
\end{abstract}

CONCLUSIONS: Intra-aneurysmal hemodynamics are altered by coils. Coil configuration might reduce its influence on intra-aneurysmal hemodynamics as the packing density increases until an insignificant influence could be achieved for high packing densities.

ABBREVIATIONS: 3DRA $=3 \mathrm{D}$ rotational angiography; ANOVA $=$ analysis of variance; $\mathrm{CFD}=$ computational fluid dynamics; DSA = digital subtraction angiography; WSS = wall shear stress lon: ntracranial aneurysms are balloon-like dilations of arteries often occurring at branching points in the circle of Willis. In most cases, these stay asymptomatic and are only diagnosed through medical imaging or after spontaneous rupture. Aneurysm rupture typically leads to subarachnoid hemorrhage producing rates of mortality of approximately 50\%. ${ }^{1}$ The existing therapeutic options seek isolating the aneurysm from the bloodstream and preventing its rupture. The most important therapies are surgical clipping and endovascular interventions, ${ }^{2}$ such as coiling and stent placement.

Coiling has been the most popular endovascular option for the last 15 years. ${ }^{1-3}$ This minimally invasive treatment consists of the placement of biocompatible metal alloy coils to block the blood flow into the aneurysm and to prevent rupture. Although coiling is associated to lower mortality and morbidity

Received December 14, 2010; accepted after revision March 5, 2011

From the Center for Computational Imaging \& Simulation Technologies in Biomedicine (H.G.M., M.K., M.-C.V.-U., I.L., A.F.F.), Information \& Communication Technologies Department, Universitat Pompeu Fabra, Barcelona, Spain; Networking Center on Biomedical Research (CIBER-BBN) (H.G.M., M.K., M.-C.V.-U., I.L., A.F.F.), Barcelona, Spain; Neuroangiografía Terapéutica J. J. Merland (E.E.V., T.S., L.G.), Hospital General de Catalunya, San Cugat del Valles, Spain; and Institució Catalana de Recerca i Estudis Avançats (A.F.F.), Barcelona, Spain.

All authors have substantially contributed to the preparation of this manuscript. Preliminary results for the virtual coiling technique and further CFD modeling previously presented at World Congress on Medical Physics and Biomedical Engineering, September 7-12, 2009, Munich, Germany.

Please address correspondence to Alejandro F. Frangi, Center for Computational Imaging \& Simulation Technologies in Biomedicine (CISTIB), Information \& Communications Technologies Department, Universitat Pompeu Fabra, c/Roc Boronat 138-E08018 Barcelona, Spain; e-mail: frangi@upf.edu

http://dx.doi.org/10.3174/ajnr.A2635 rates compared with surgical clipping, ${ }^{2}$ its outcome is not always predictable. ${ }^{1-5}$ Packing density is thought to have a strong influence in postcoiling outcome, and usually the aneurysms are packed as dense as possible. ${ }^{6-8}$ Packing density is defined as the ratio between the inserted coils and aneurysm volume.

Moreover, the success of coiling is intimately related to the biologic responses to hemodynamics inside the aneurysm. ${ }^{9}$ Certainly, CFD is an effective and well-accepted technique to investigate intravascular hemodynamics in silico. ${ }^{10}$ However, the use of this technique in the presence of endovascular coils is still challenging, due to the geometric complexity of the devices, their small size (diameters of $0.010-0.015$ inch), ${ }^{11}$ and their unpredictable distribution inside the aneurysm.

To simulate the hemodynamics in coiled aneurysm geometries, researchers have modeled explicitly the coils, including straight ${ }^{12}$ or helical cylinders, ${ }^{13}$ big hollow spheres, ${ }^{14}$ or small overlapping spheres along the axis of curved filaments. ${ }^{15}$ Other approaches assume that coils act as a porous medium inside the aneurysm. ${ }^{16-19}$ Most of these studies have taken into account the influence of packing density on the hemodynamics. However, little is known about how coil configuration, ie, the arrangement of coils inside the aneurysm, could affect the blood flow. Recently, an in vitro experiment has studied qualitatively the influence of both coil configuration and packing density, but only on near-wall flow. ${ }^{20}$ In addition, a CFDbased study on an idealized sidewall aneurysm geometry found that the orientation of helical coils has influence on intra-aneurysmal hemodynamics. ${ }^{13}$

The purpose of this work is to qualitatively and quantita- 


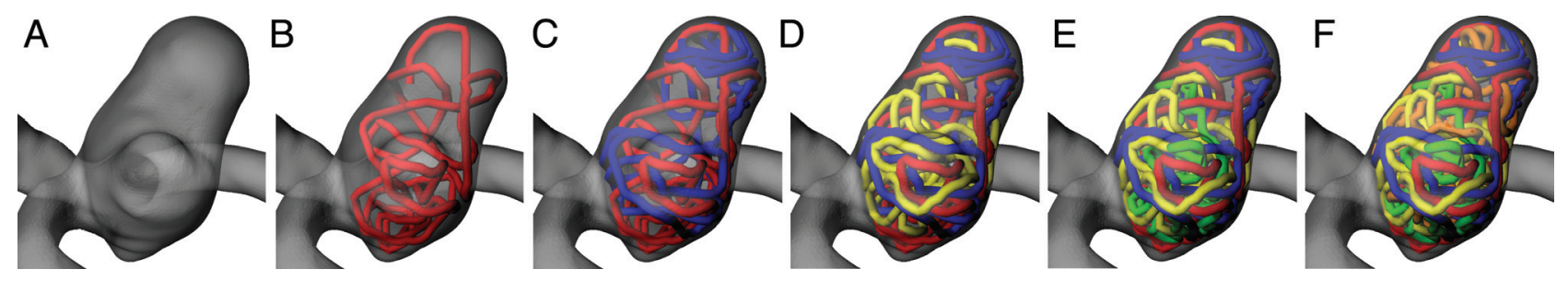

Fig 1. Results of virtual coiling for case 2. Untreated model $(A)$ and treated models corresponding to coil packing densities of $5.6 \%(B), 11.2 \%(C), 16.7 \%(D), 22.2 \%(E)$, and $30.0 \%(F)$.

tively assess the influence of coil configuration and packing density on intra-aneurysmal hemodynamics. Three patientspecific aneurysm geometries were virtually treated with coils, and CFD simulations were performed in both untreated and treated geometries. The main hypothesis behind our CFD experiments was that intra-aneurysmal hemodynamics strongly depend on the mechanical resistance produced by the coils as was reported in the literature. ${ }^{21}$

\section{Materials and Methods}

\section{Vascular Models}

Three patient-specific aneurysms at different locations in the human brain vasculature were chosen. The locations for cases 1,2 , and 3 were respectively left internal carotid sinus, right middle cerebral artery bifurcation, and basilar tip. All 3 cases were selected for treatment with endovascular coils. 3DRA images of the aneurysms were obtained by using an Integris System (Philips Healthcare, Best, the Netherlands). The size of the images in voxels was $256 \times 256 \times 256$, with an FOV of $134.95 \mathrm{~mm}$. A 3D representation of the luminal surfaces of the vessels and aneurysms was obtained after segmentation of the medical images by using an iso-intensity surface extraction approach. Afterward, the extracted 3D geometries were cleaned, cropped, and smoothed. $^{22}$

\section{Virtual Coiling}

The 3 cases were treated by using a virtual coiling technique. ${ }^{23,24}$ This technique was designed to place coil models inside aneurysm geometries. The coils are characterized by their geometry, namely, wire diameter and length.

Each coil is progressively placed by advancing its tip, which starts from an initial position inside the aneurysm. The coil tip motion is constrained by the aneurysm dome and its ostium. If the tip of the coil is close to either the surface of the aneurysm or another coil previously inserted, the tip smoothly changes its direction to remain inside the aneurysm and to avoid collision with the other coils. When the coil tip cannot move further because it is blocked by other coils previously placed, the virtual coiling technique partially retracts and redirects the tip toward another region. For a more detailed explanation of the method, see the Appendix.

The technique sequentially places the coils inside the aneurysms until the desired packing density is achieved. As a result, a plausible representation of the distribution of the coils is produced as it could be obtained after a real intervention (Fig 1).

This virtual coiling technique was used in our 3 cases. Five coil packing densities were produced (Table 1). Packing densities were calculated from the volumes of the 3D geometries. Also, for each packing density, 3 coil configurations were generated and labeled as I, II, and III. In this way, the influence of geometric dissimilarities due to the coil configuration on intra-aneurysmal hemodynamics could be evaluated.

\begin{tabular}{|c|c|c|c|c|c|}
\hline $\begin{array}{l}\text { Case } \\
\text { No. }\end{array}$ & $\begin{array}{c}\text { Volume of } \\
\text { Aneurysm } \\
\text { Region } \\
\left(\mathrm{mm}^{3}\right)\end{array}$ & $\begin{array}{c}\text { Coil } \\
\text { Diameter } \\
(\mathrm{mm})\end{array}$ & $\begin{array}{l}\text { Coil } \\
\text { Length } \\
\text { (cm) }\end{array}$ & $\begin{array}{l}\text { Coil } \\
\text { Shape }\end{array}$ & $\begin{array}{l}\text { Coil Packing } \\
\text { Density (\%) }\end{array}$ \\
\hline 1 & 107 & 0.304 & $5-30$ & Straight & $6.8,13.6,20.0,25.0,31.5$ \\
\hline 2 & 423 & 0.457 & $4-30$ & Straight & $5.6,11.2,16.7,22.2,30.0$ \\
\hline 3 & 635 & 0.457 & $4-30$ & Straight & $7.0,14.0,21.0,27.5,32.0$ \\
\hline
\end{tabular}

\section{Hemodynamic Modeling}

To solve the governing equations of fluid flow, body-fitted unstructured tetrahedral meshes were generated with the vascular geometries and coils by using the commercial software ANSYS ICEM CFD version 11 (Ansys, Canonsburg, Pennsylvania). In the treated models, the volumetric mesh near the coils was refined, and a convergence test was performed to obtain mesh-independent CFD simulations. The test criterion was set to be below $5 \%$ of differences of the averaged WSS at the aneurysm between consecutive mesh refinements. Average WSS was found to fulfill this criterion when the coil cross-section perimeter was enclosed with approximately 10 mesh elements.

These volumetric meshes were exported in ANSYS CFX version 11 (Ansys) for the CFD simulations. Blood was modeled as a Newtonian fluid with viscosity of $0.00375 \mathrm{~Pa} \times$ s. Flow was assumed to be laminar and incompressible (blood density of $1060 \mathrm{~kg} / \mathrm{m}^{3}$ ). Vessel wall and coil surfaces were imposed to be rigid with no-slip boundary condition on the flow. At the inlet of the models, a parabolic velocity profile (spatial condition) was imposed as well as a physiologic blood flow waveform (temporal condition) from a normal individual. These flow waveforms were scaled to ensure a time-averaged Reynolds number of 300 at the inlet of each model. At the outlets, a traction-free boundary condition was applied.

Two entire cardiac cycles were simulated, and the results from the second cycle were analyzed as time-averaged, and at peak systole and end diastole. The blood flow velocity, the average WSS, and the areas of low $(<0.4 \mathrm{~Pa})$ and high WSS $(>1.5 \mathrm{~Pa})$ were evaluated in the aneurysm. These WSS thresholds were selected because of their association with pathologic states of the arterial wall. ${ }^{25}$

\section{Statistical Evaluation}

ANOVA tests were performed to evaluate the relative effect of the coil configuration on the intra-aneurysmal hemodynamics at different packing densities. The results for an entire cardiac cycle were taken into account as samples for the tests. Before applying the tests, the hypotheses of normal distribution, homogeneity of the variance, and independence were confirmed for each sample.

\section{Results}

\section{Qualitative Findings}

Figure 2 depicts velocity magnitudes at peak systole on a fixed aneurysm cross-section for case 2. Packing densities are shown 

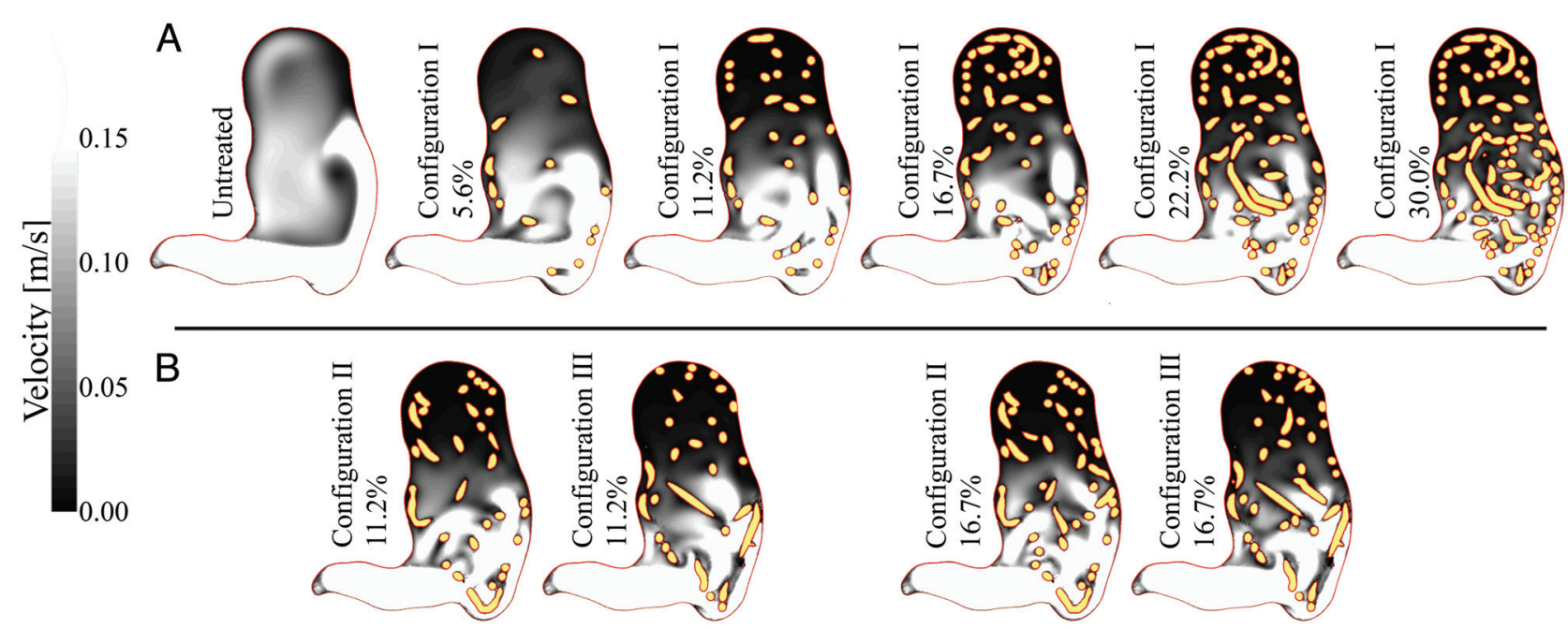

Fig 2. Blood flow velocity magnitudes in gray-scale for an aneurysm cross-section at peak systole for case 2 . $A$, Changes in the velocity are presented with a fixed coil configuration and increasing packing density. B, Two coil configurations are compared for packing densities of $11.2 \%$ and $16.7 \%$. The coils are presented in yellow and the percentages represent the packing densities achieved for each model.
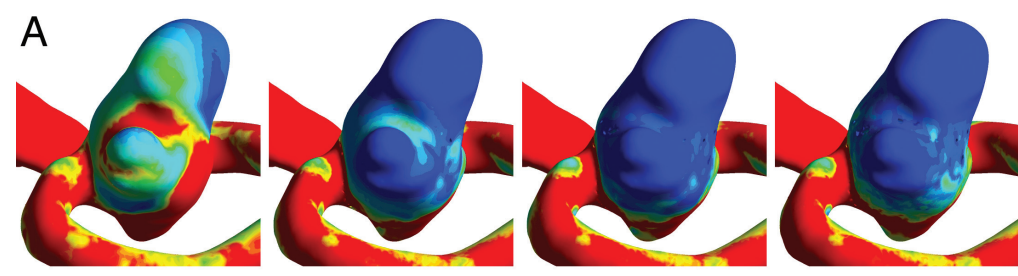

WSS

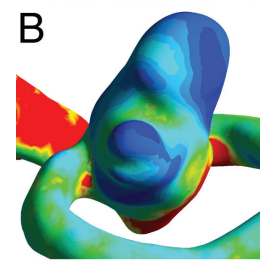

Untreated
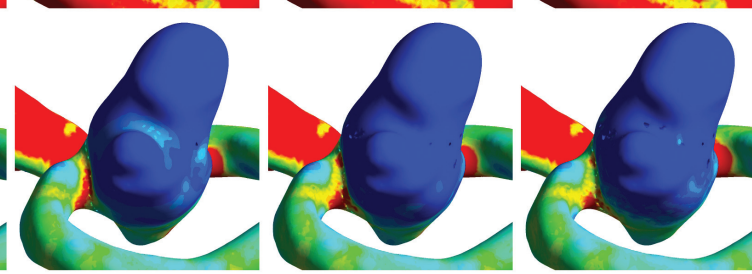

$(\mathrm{Pa})$

Fig 3. WSS distributions for the untreated and several treated models for case 2, calculated at peak systole $(A)$ and end diastole $(B)$.

in Fig $2 A$, and Fig $2 B$ compares different coil configurations. The untreated aneurysms showed high-velocity regions with strong flow impingement on the distal wall and a vortical flow structure inside the aneurysm. For packing densities $<11.2 \%$, inflow was disturbed and aneurysmal flow velocity was decreased, especially at the aneurysm fundus. As the number of coils increased, a progressive reduction in the flow velocity inside the aneurysm was observed. Nonetheless, small additional velocity reductions were associated with coil packing densities $>22.2 \%$. Furthermore, Fig $2 B$ shows how 2 coil configurations for case 2 were able to visually induce substantial differences on the flow fields at a low packing density, such as $11.2 \%$.

Visualization of WSS distributions at peak systole and enddiastole are presented in Fig $3 A$ and $B$, respectively. Here, simulations for case 2 are shown with and without coils. A reduction of WSS was already observed for a low packing density of $5.6 \%$, whereas above $16.7 \%$, a stable low WSS throughout the cardiac cycle was found at the aneurysm dome. A remarkably high WSS at peak systole near the aneurysms' neck were also observed. Similar WSS distributions as well as flow structures and velocity reduction trends were visualized for cases 1 and 3 .

\section{Quantitative Findings}

Time-averaged velocity reduction inside the aneurysm based on the untreated model was computed for the different coil configurations and packing densities (Fig 4A). The velocity reduction was almost independent of the coil configuration for packing densities $>25 \%$. In general, velocity reduction increased as packing density rose. For high packing densities (near 30\%), the coils induced a maximum velocity reduction of approximately $70 \%$ for case $1,65 \%$ for case 2 , and almost $90 \%$ for case 3 .

Figure $4 B$ shows time-averaged aneurysmal WSS. For all cases, a reduction in WSS was found after coiling, even for those with lower packing densities. A progressive decrease of the average WSS was observed as packing density increased. For low packing densities $(<10 \%)$, a large variability in the average WSS was induced by the coil configuration, especially for case 2, with $5.6 \%$ of packing density (mean WSS of $1.65 \mathrm{~Pa}$ and SD of $0.4 \mathrm{~Pa}$ ).

To analyze the evolution of the WSS in a cardiac cycle, the areas of low $(<0.4 \mathrm{~Pa})$ and high $(>1.5 \mathrm{~Pa})$ WSS were computed for each aneurysm. In Fig 5, these areas were presented for 3 CFD models of case 2, including the untreated model (Fig 

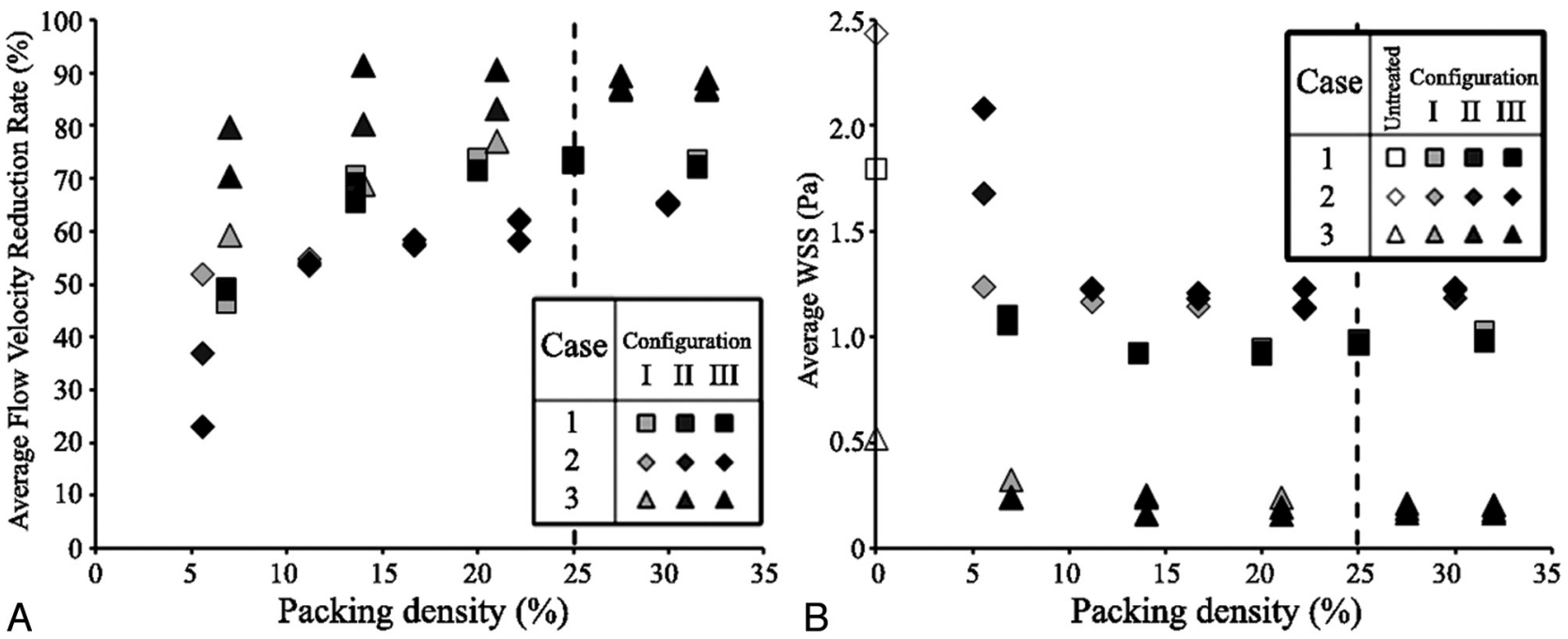

Fig 4. $A$, Time-averaged intra-aneurysmal mean flow velocity reduction rate. $B$, Time-averaged WSS in the aneurysm dome.
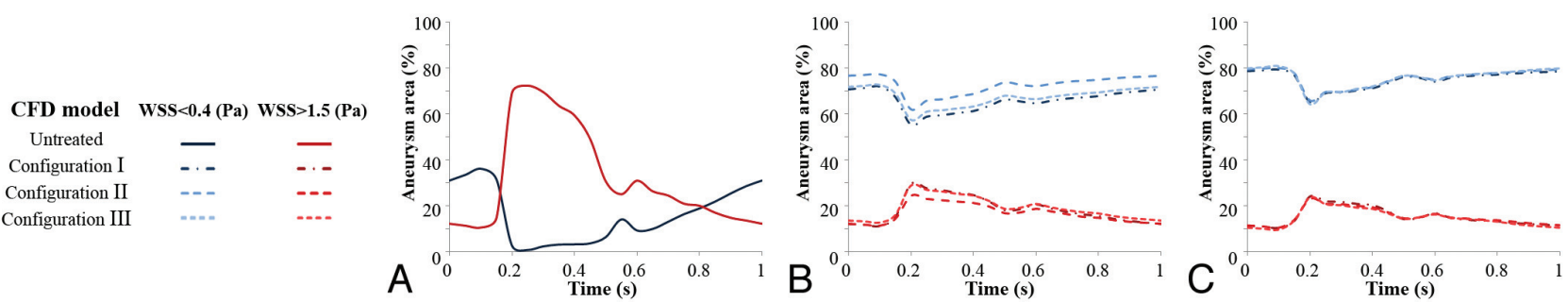

Fig 5. Variation of the aneurysm areas with low and high WSS expressed as a percentage of the whole aneurysm dome during one cardiac cycle for case 2. $A$, Untreated model. $B$ and C, treated models with packing densities of $22.2 \%$ and $30.0 \%$, respectively.

\begin{tabular}{|c|c|c|c|c|c|}
\hline \multirow{4}{*}{$\begin{array}{l}\text { Case } \\
\text { No. }\end{array}$} & \multirow{4}{*}{$\begin{array}{c}\text { Packing } \\
\text { Density } \\
(\%)\end{array}$} & \multicolumn{4}{|c|}{$P$ Value Obtained from ANOVA Tests for } \\
\hline & & Velocity & & Low & High \\
\hline & & Reduction & Average & WSS & WSS \\
\hline & & Rate & WSS & Area & Area \\
\hline \multirow[t]{3}{*}{1} & 20.0 & $.001^{*}$ & .981 & .245 & .834 \\
\hline & 25.0 & .064 & .988 & .833 & .886 \\
\hline & 31.5 & .127 & .941 & .269 & .723 \\
\hline \multirow[t]{2}{*}{2} & 22.2 & $.000^{*}$ & .927 & $.000^{*}$ & .309 \\
\hline & 30.0 & .869 & .981 & .181 & .956 \\
\hline \multirow[t]{3}{*}{3} & 21.0 & $.000^{*}$ & .373 & $.002^{*}$ & .163 \\
\hline & 27.5 & .077 & .601 & .757 & .583 \\
\hline & 32.0 & .155 & .655 & .760 & .552 \\
\hline
\end{tabular}

* Means significantly different due to coil configuration $(P<.01)$.

$5 A)$. A clear difference in these areas is visualized between the simulations with and without coils. After coiling, low WSS areas increased and reached values of approximately $75 \%$ for case $1,60 \%$ for case $2 \%$, and $95 \%$ for case 3 . The high WSS areas decreased and were approximately $20 \%$ for cases 1 and 2, and $<10 \%$ for case 3 . Also, there were noticeable differences in the WSS areas due to the coil configuration for a packing density $<22.2 \%$ (Fig $5 B$ ), especially near peak systole. However, these differences were less evident as packing density increased (Fig 5C).

\section{Analysis of Statistical Data}

The ANOVA test results (Table 2) showed that there were nonsignificant differences $(P>.01)$ on the average WSS and high WSS areas due to the coil configuration when packing density was $>20 \%$. However, there were significant differences $(P<.01)$ in the velocity reduction and low WSS areas when the packing density was $<22.2 \%$ in all 3 cases (case 2 had the highest packing density). The only exception to this result was for the low WSS areas in case 1, in which the differences induced by the coil configuration were negligible. Table 2 also shows that, for the studied cases, the hemodynamic variables derived from different coil configurations were nonsignificant for packing densities of approximately $30 \%$.

\section{Discussion}

The present study aims at elucidating how packing density and coil configuration might influence the intra-aneurysmal hemodynamics. Four main findings were obtained from our numeric modeling and data analyses. First, the intra-aneurysmal velocity and WSS decreased as packing density increased. Second, the influence of coil configuration on intra-aneurysmal hemodynamics proved to be negligible for elevated packing densities (near 30\%). However for lower packing densities, this influence was larger and statistically significant. Third, aneurysmal flow velocity was reduced by $>50 \%$ after the insertion of the first coils (packing density $<12 \%$ ). Fourth, evidence was found that the blood flow waveform is damped near the aneurysm wall as packing density increases, which was reflected in the flattening of the areas with low and high WSS.

These findings were obtained by qualitative observations, analyses of quantitative data, and statistical evaluations of our CFD simulations. The first hemodynamic variable analyzed 
was blood flow velocity inside the aneurysm. In Fig 2, a progressive reduction of the velocity inside the aneurysm was observed as packing density increased. This finding is consistent with previous CFD-based studies and in vitro experiments. ${ }^{13,15,19,20,26}$ Particular flow patterns were visualized due to the coil configuration (Fig $2 B$ ), which are associated with the special location of the coils at the aneurysm ostium. Specific flow characteristics also were reported by an in vivo study, in which short and disturbed streamlines were found near the aneurysm wall. ${ }^{20}$

The second evaluated hemodynamic variable was average WSS. In Fig 3, a stable (systole and diastole) low WSS distribution (blue areas) was found as packing densities were $>16.7 \%$. This was mainly observed in the aneurysm fundus. Furthermore, high WSS (red areas) was localized at the aneurysm neck, which is associated with the blockage and reflection of the flow stream due to the coils. These WSS distributions were previously observed in a CFD-based study modeling the coils as a porous medium. ${ }^{18}$ Also, the reduction of average WSS as packing density increased was noticeable in Fig 3 by the increment of the blue area.

The third hemodynamic variable was the intra-aneurysmal velocity reduction. In Fig $4 A$, the previous qualitative observations were confirmed as for the influence of packing density and coil configuration on the local hemodynamics. The efficiency of the coils reducing the intra-aneurysmal flow velocity depends on coil configuration and packing density as is visualized among cases. Furthermore, the dependency of the velocity reduction on coil configuration was lower for high packing densities $(>25 \%)$.

For our 3 cases, the insertion of the first coils (packing densities $<12 \%$ ) produced the most important velocity reduction $(>50 \%)$ inside the aneurysm. However, more variability of the velocity reduction was induced by low packing densities (see cases 2 and 3 in Fig 4A). In general, an asymptotic trend of the velocity reduction was observed as packing density increased. In other words, the last coils have a smaller role in the velocity reduction inside the aneurysm. Additionally, the maximum velocity reduction observed in Fig $4 A$ for our cases (70\%-90\%), corresponds well to previous CFDbased studies $^{18}$ and in vivo animal experiments. ${ }^{27}$

The last hemodynamic variables studied were the aneurysmal areas of low $(<0.4 \mathrm{~Pa})$ and high $(>1.5 \mathrm{~Pa})$ WSS, presented in Fig 5. Here, the high/low WSS areas were damped by the coils, suggesting that the hemodynamics near the wall tend to be steady as packing density increases. However, the damping was strongly related to packing density. Furthermore, most of the variations in WSS areas happened near the aneurysm neck (Fig 3), thereby WSS at the aneurysm dome was almost timeinvariant. This steady hemodynamic condition at the aneurysm wall combined with the previously mentioned low-shear stresses and low-velocity flow might be favorable hemodynamic conditions to isolate the aneurysm from the bloodstream. ${ }^{9,28}$ In addition, it was observed how coil configuration reduces its influence on hemodynamics when packing density increases (Fig 5).

The statistical tests were performed to study the variability induced by the coil configuration as packing density increased on the studied hemodynamic variables. We found that coil configuration has an influence on intra-aneurysmal hemody- namics when packing density is low. However, the hemodynamic dependence on coil configuration was reduced as packing density increased. This dependence was statistically nonsignificant $(P>.01)$ for packing densities of approximately $30 \%$.

From our results, we observe that intra-aneurysmal hemodynamics tend to stabilize as packing density increases and becomes independent of coil configuration. However, the reported packing densities do not ensure the success of coiling, mainly because the required hemodynamic conditions to warranty aneurysm occlusion by coiling are unknown. Also, the presented results are based on the flow waveforms imposed as boundary conditions, which were physiologic and measured under rest condition but neither patient-specific nor generic. As it has been found that local hemodynamic forces are associated with heart rate and aneurysm geometry, ${ }^{29}$ further investigation is required to generalize or extrapolate our findings to other physiologic flow waveforms (different heart rate, flow rate, or waveform shape) and different vascular morphologies.

Our results have proved to be comparable to previous CFD-based studies with coils, but our virtual coiling technique presents some advantages over these. The first advantage is that our technique has the potential of becoming relevant in clinical practice. It is able to reproduce the wide range of packing densities as it is obtained in real interventions ${ }^{6-8}$ and in vitro experiments ${ }^{30}$ with coils. Furthermore, it considers the geometric specifications of coils, such as wire diameter and length, and can be used in patient-specific geometries. The technique uses medical images before treatment to extract the $3 \mathrm{D}$ representation of the vasculature, and the manual definition of the ostium to constrain the space occupied by the coil models provides a tool for preoperatory hemodynamic assessment. In addition, the use of images after treatment allows us to extract similar regions to insert the coils as the regions visualized with medical images, which provides postoperative hemodynamic assessment.

The other advantage is the explicit modeling of the coils, which allowed us to investigate the influence of coil configuration and packing density on the intra-aneurysmal hemodynamics. Certainly, packing density can be modeled with a porous medium approach by setting the porosity, ${ }^{16,18,19}$ but the simulation of different coil configurations is not trivial due to the assumptions of homogeneity and isotropy of the medium. In contrast, a study by Cebral and Löhner ${ }^{15}$ proposed an explicit modeling of the coils for further CFD analysis. However, it is not clear the packing densities that can be achieved with the generation of arbitrary objects and more specifically, the coil distribution near the aneurysm walls. Finally, the helical coils presented by Schirmer and Malek ${ }^{13}$ in an idealized aneurysm geometry are fully user-dependent and did not achieve high packing densities.

Despite these advantages and our interesting findings, this work has some limitations. One limitation is that the coils were assumed as rigid bodies and their motion was not allowed in our CFD simulations, which means that the coils were not affected by the flow. Although this assumption considerably simplifies the numeric solution, coil deformation as well as a more precise interaction with the flow should be taken into account to investigate other relevant issues related to this therapy, such as coil compaction. Also, another limita- 
tion is that the vessel and aneurysm walls were considered as rigid bodies due to the lack of information regarding wall thickness and mechanical properties of the tissue. Nevertheless, such choice is supported by several CFD-based studies that assumed the rigid wall condition and claimed that this effect is minor compared with others, such as lumen morphology. ${ }^{15,16,18,19,31}$

A CFD simulation able to assess the success or failure of coiling an aneurysm should consider the thrombus formation inside the aneurysm. In this work, thrombus formation was not tackled because efforts were mainly focused on evaluating the hemodynamic resistance of the coils as these have been found to be relevant experimentally. ${ }^{21}$ In addition, thrombus formation has been related to hemodynamics and blood viscosity. ${ }^{9}$ Our study was limited to the use of a Newtonian model for blood viscosity, and further investigation is required to establish the influence of the non-Newtonian behavior of blood on the hemodynamics of coiled aneurysms. The assumption of Newtonian flow is justified because it has been reported to be as good as non-Newtonian models in similar studies in arteries with aneurysms compared with the uncertainties introduced by geometric models. ${ }^{31}$ This aspect also has been recently used to assess the effect of flow diverters on intra-aneurysmal hemodynamics. ${ }^{32}$ However, the consideration of a non-Newtonian model opens questions such as which non-Newtonian model (eg, Casson, Herschel-Bulkley, or Power law) represents more accurately the behavior of blood and why, what are the differences and similarities compared with a Newtonian model, and whether these differences are meaningful.

\section{Conclusions}

We have studied the influence of coiling treatment on intraaneurysmal hemodynamics in 3 patient-specific aneurysm geometries by using a virtual coiling technique and CFD modeling. Four main findings were extracted from our results. First, coils attenuate blood flow velocity and WSS with increasing packing density. Second, the influence of coil configuration on intra-aneurysmal hemodynamics is reduced with increasing packing density, such that no significant influence $(P>.01)$ is found for packing densities of $30 \%$. Third, the first inserted coils (packing density $<12 \%$ ) cause $>50 \%$ of the velocity reduction inside the aneurysm. Finally, a damping effect is observed on WSS areas due to the coils, which creates a stable hemodynamic condition inside the aneurysm.

\section{Appendix}

\section{Virtual Coiling Technique}

The virtual coiling technique uses a dynamic path planning approach to insert one-by-one the coils inside a closed geometry (in this case, aneurysm geometry was defined by its wall and ostium). Inserting a coil means consecutively selecting the next position of the coil tip. This position is selected among a set of candidate positions around the tip by evaluating a potential field $\phi$ for each of them. The candidate position with the lowest $\phi$ is chosen as the next tip position. In this way, the algorithm inserts a coil (creates a path) by advancing the coil tip.
The potential field $\phi$ has 4 rules (or subpotential fields) that mimic the observed behavior of coils during their placement.

$$
\phi=\phi_{A}+\phi_{C}+\phi_{D}+\phi_{P B}
$$

The first rule $\phi_{A}$ constrains the region where the coil tip is allowed to move, and it is limited by the aneurysm wall and the ostium. It ensures that the coil tip remains inside the aneurysm, and it is defined as 0 inside the aneurysm and 1 elsewhere.

The second rule $\phi_{C}$ avoids the collision between coils and keeps the coil tip away from highly dense coiled areas. The rule $\phi_{C}$ takes values between 0 and 1 , being 1 when the evaluated position is occupied by a previously inserted coil.

The third rule $\phi_{D}$ considers the deformation of the coils. Basically, the larger the angle of the evaluated candidate is, compared with the previous direction, the larger $\phi_{D}$ will be. This rule presents values between 0 and 0.5 , being 0 for an angle equal to 0 and 0.5 when the angle of the candidate position is $\pi$.

Finally, the rule $\phi_{P B}$ is meant to solve the situation where the tip is trapped by the aneurysm wall, other coil(s), or both, which means that all candidate locations have $\phi \geq 1$ (deadend). In this situation, the algorithm pulls back and advances the coil tip in a different direction.

To initialize a coil, a set of candidate initial positions is created and the minimizing $\phi_{C}+\phi_{D}$ is selected. For the first inserted coil, only $\phi_{D}$ is considered, which is compared with a direction provided by the user. This guarantees that by changing this initial parameter, the coil configuration is changed.

\section{Virtual Coiling Technique Assessment}

To assess the effect of the virtual coiling technique on hemodynamics in comparison with the real coils, ${ }^{24}$ a CFD model was generated from medical images available for a coiled aneurysm. The aneurysm was located at the right middle cerebral artery, and treated with a $43 \%$ packing density.

To construct the CFD model, a 3DRA image of the patient was segmented, cropped, cleaned, and smoothed to obtain the vascular model of the aneurysm and parent artery. Then, the virtual coiling technique was used to insert in the vascular model the same number of coils with the same geometric characteristics as in the real treatment. To set the boundary conditions of the CFD model, pseudopatient-specific conditions were imposed. A time-intensity curve was extracted from the DSA image sequence at the location of the model inlet. This curve was calculated as the average pixel intensity in a region near the inlet for each time instant. This information was used as boundary condition to solve an advection-diffusion equation to calculate the transport of a contrast material, modeled as a mass-less scalar.

After the CFD simulation, a set of virtual angiographic images were created. These virtual images were qualitatively compared with the DSA image sequence of the patient and a good agreement between these was found. Time-intensity curves also were measured at several control regions along the vasculature on the DSA and virtual images (CFD-based) for a quantitative comparison, including locations downstream of the coiled aneurysm. The comparison of both time-intensity curves showed an excellent correlation in all the evaluated control regions. 
Disclosures: Hernán Morales, Research Support (including provision of equipment or materia/s): Universitat Pompeu Fabra/CISTIB, Details: This work was partially supported within the CENIT program, as part of CDTEAM and CVREMOD projects funded by the Spanish CDTI and partly within the framework of the @ neurIST Project (IST-2005-027703), which is cofinanced by the European Commission within the IST Program of the Sixth Framework Program; Minsuok Kim, Research Support (including provision of equipment or materials): Spanish CDTI, European Commission, Details: This work was partially supported within the CENIT program, as part of CDTEAM and CVREMOD projects funded by the Spanish CDTI and partly within the framework of the @neurlST Project (IST-2005-027703), which is cofinanced by the European Commission within the IST Program of the Sixth Framework Program; Ignacio Larrabide, Research Support (including provision of equipment or materials): CIBER-BBN, Details: Centro de Investigación Biomédica en Red en Bioingeniería, Biomateriales y Nanomedicina. This researcher is funded by this institution; Alejandro Frangi, Research Support (including provision of equipment or materials): ANSYS, Details: The company provides us with free software licenses for numeric simulation of flow dynamics. This researcher does not anticipate any conflict of interest.

\section{References}

1. van Gijn J, Rinkel GJ. Subarachnoid haemorrhage: diagnosis, causes and management. Brain 2001;124:249-78

2. Molyneux AJ, Kerr RS, Yu LM, et al. International subarachnoid aneurysm trial (ISAT) of neurosurgical clipping versus endovascular coiling in 2143 patients with ruptured intracranial aneurysms: a randomised comparison of effects on survival, dependency, seizures, rebleeding, subgroups, and aneurysm occlusion. Lancet 2005;366:809-17

3. Henkes H, Fischer S, Weber W, et al. Endovascular coil occlusion of 1811 intracranial aneurysms: early angiographic and clinical results. Neurosurgery 2004;54:268-80; discussion 80-85

4. Park SH, Lee CY, Yim MB. The merits of endovascular coil surgery for patients with unruptured intracranial aneurysms. J Korean Neurosurg Soc 2008;43: $270-74$

5. Raymond J, Guilbert F, Weill A, et al. Long-term angiographic recurrences after selective endovascular treatment of aneurysms with detachable coils. Stroke 2003;34:1398-403

6. Kawanabe Y, Sadato A, Taki W, et al. Endovascular occlusion of intracranial aneurysms with Guglielmi detachable coils: correlation between coil packing density and coil compaction. Acta Neurochir (Wien) 2001;143:451-55

7. Sluzewski M, van Rooij WJ, Slob MJ, et al. Relation between aneurysm volume, packing, and compaction in 145 cerebral aneurysms treated with coils. Radiology 2004;231:653-58

8. Tamatani S, Ito Y, Abe H, et al. Evaluation of the stability of aneurysms after embolization using detachable coils: correlation between stability of aneurysms and embolized volume of aneurysms. AJNR Am J Neuroradiol 2002;23: 762-67

9. Wootton DM, Ku DN. Fluid mechanics of vascular systems, diseases, and thrombosis. Annu Rev Biomed Eng 2003;1:299-329

10. Alnaes MS, Isaksen J, Mardal KA, et al. Computation of hemodynamics in the circle of Willis. Stroke 2007;38:2500-05

11. White JB, Ken CG, Cloft HJ, et al. Coils in a nutshell: a review of coil physical properties. AJNR Am J Neuroradiol 2008;29:1242-46

12. Narracott A, Smith S, Lawford P, et al. Development and validation of models for the investigation of blood clotting in idealized stenoses and cerebral aneurysms. J Artif Organs 2005;8:56-62

13. Schirmer CM, Malek AM. Critical influence of framing coil orientation on intra-aneurysmal and neck region hemodynamics in a sidewall aneurysm model. Neurosurgery 2010;67:1692-702

14. Byun HS, Rhee K. CFD modeling of blood flow following coil embolization of aneurysms. Med Eng Phys 2004;26:755-61
15. Cebral JR, Löhner R. Efficient simulation of blood flow past complex endovascular devices using an adaptive embedding technique. IEEE Trans Med Imaging 2005;24:468-76

16. Cha KS, Balaras E, Lieber BB, et al. Modeling the interaction of coils with the local blood flow after coil embolization of intracranial aneurysms. J Biomech Eng 2007;129:873-79

17. Groden C, Eckert B, Ries T, et al. Angiographic follow-up of vertebrobasilar artery aneurysms treated with detachable coils. Neuroradiology 2003;45: 435-40

18. Jou L-D, Saloner D, Higashida R. Determining intra-aneurysmal flow for coiled cerebral aneurysm with digital fluoroscopy. Biomed Eng Appl Basis Comm 2004;16:6

19. Kakalis NM, Mitsos AP, Byrne JV, et al. The haemodynamics of endovascular aneurysm treatment: a computational modelling approach for estimating the influence of multiple coil deployment. IEEE Trans Med Imaging 2008;27: $814-24$

20. Goubergrits L, Thamsen B, Berthe A, et al. In vitro study of near-wall flow in a cerebral aneurysm model with and without coils. AJNR Am J Neuroradiol 2010 31:1521-28

21. Sorteberg A, Sorteberg W, Aagaard BD, et al. Hemodynamic versus hydrodynamic effects of Guglielmi detachable coils on intra-aneurysmal pressure and flow at varying pulse rate and systemic pressure. AJNR Am J Neuroradiol 2004; 25:1049-57

22. Villa-Uriol MC, Larrabide I, Geers AJ, et al. AngioLab: integrated technology for patient-specific management of intracranial aneurysms. Conf Proc IEEE Eng Med Biol Soc 2010;1:6801-04

23. Morales HG, Kim M, Villa-Uriol M-C, et al. Influence of coil packing rate and configuration on intracranial aneurysm hemodynamics. World Congress on Medical Physics and Biomedical Engineering, September 7-12, 2009, Munich, Germany 2009;25/4

24. Morales HG, Larrabide I, Villa-Uriol M-C, et al. Towards the validation of a virtual coiling technique using a real versus a simulated bolus injection. $\mathrm{Sec}$ ond International Conference on Computational \& Mathematical Biomedical Engineering, March 30-April 1, 2011, Washington, DC; 512-15

25. Malek AM, Alper SL, Izumo S. Hemodynamic shear stress and its role in atherosclerosis. JAMA 1999;282:2035-42

26. Babiker MH, Gonzalez LF, Albuquerque F, et al. Quantitative effects of coil packing density on cerebral aneurysm fluid dynamics: an in vitro steady flow study. Ann Biomed Eng 2010;38:2293-301

27. Sorteberg A, Sorteberg W, Rappe A, et al. Effect of Guglielmi detachable coils on intraaneurysmal flow: experimental study in canines. AJNR Am J Neuroradiol 2002;23:288-94

28. Mori K, Nakao Y, Horinaka N, et al. Cerebral aneurysm regrowth and coil unraveling after incomplete Guglielmi detachable coil embolization: serial angiographical and histological findings. Neurol Med Chir (Tokyo) 2003;43: 293-97

29. Jiang J, Strother C. Computational fluid dynamics simulations of intracranial aneurysms at varying heart rates: a "patient-specific" study. J Biomech Eng 2009;131:091001

30. Piotin M, Mandai S, Murphy KJ, et al. Dense packing of cerebral aneurysms: an in vitro study with detachable platinum coils. AJNR Am J Neuroradiol 2000;21: 757-60

31. Cebral JR, Castro MA, Appanaboyina S, et al. Efficient pipeline for image-based patient-specific analysis of cerebral aneurysm hemodynamics: technique and sensitivity. IEEE Trans Med Imaging 2005;24:457-67

32. Cebral JR, Mut F, Raschi M, et al. Aneurysm rupture following treatment with flow-diverting stents: computational hemodynamics analysis of treatment. AJNR Am J Neuroradiol 2011;32:27-33 